\title{
Feldeffektbasierte pH-Sensoren für die Überwachung von sauren Grubenwässern
}

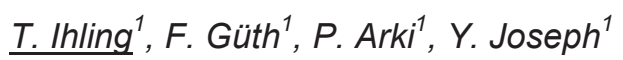 \\ ${ }^{1}$ Institut für Elektronik- und Sensormaterialien, TU Bergakademie Freiberg, Freiberg, Deutschland \\ Kontakt: Thomas.Ihling@esm.tu-freiberg.de
}

\section{Einleitung}

Die messtechnische Überwachung und Behandlung von sauren Grubenwässern (engl. Acid Mine Drainage, AMD) spielt eine wesentliche Rolle im Bereich des Umweltschutzes, sowie im Korrosionsschutz von Bauwerken und Geräten. Saure Grubenwässer entstehen beim Eisen- und Kohlebergbau. Dort kommt es beim Rohstoffabbau durch die Freilegung von Sulfidmineralien (wie Pyrit) beim Kontakt mit dem ins Bergwerk einfließenden Wasser und Sauerstoff aus der Luft zur Reaktion zu sauren Grubenwässern [1].

$$
\mathrm{FeS}_{2}+3,75 \mathrm{O}_{2}+0,5 \mathrm{H}_{2} \mathrm{O} \rightarrow \mathrm{Fe}^{3+}+2 \mathrm{SO}_{4}{ }^{2-}+\mathrm{H}^{+}
$$

Die durch den Bergbau freigelegten Eisensulfidmineralien reagieren dabei zu Schwefelsäure und lösen $\mathrm{Fe}^{2+}$-Ionen aus dem Felsgestein. Die $\mathrm{Fe}^{2+}$-Ionen und der Schwefel werden durch spezielle Bakterien und Archaeen zu Fe ${ }^{3+}$ Ionen und Schwefelsäure oxidiert [1]. Die gelösten $\mathrm{Fe}^{3+}$ Ionen färben das ins Bergwerk eindringende Grubenwasser rot-braun und gelangen früher oder später aus dem Bergwerk in Strömungsgewässer [2]. Die freigesetzten $\mathrm{H}^{+}$Ionen führen zudem zu einer sauren $\mathrm{pH}$-Wertänderung. Aufgrund der unterschiedlichen Lage der Stollen kommt es zudem zu unterschiedlichen Temperaturen, Durchflussmengen, sowie geochemischen Milieubedingungen mit verschiedenen mehrfachionenangereicherten Grubenwässern [3] [4]. Beispielsweise sind die Hauptinhaltsstoffe der Grubenwässer des Lehr- und Forschungsbergwerks der TU Bergakademie Freiberg "Reiche Zeche“ nach [5]: $\mathrm{HCO}_{3}{ }^{-}, \mathrm{Cl}^{-}, \mathrm{NO}_{2}^{-}, \mathrm{NO}_{3}^{-}, \mathrm{SO}_{4}{ }^{2-}, \mathrm{Na}^{+}, \mathrm{K}^{+}, \mathrm{Ca}^{2+}$, $\mathrm{Mg}^{2+}, \mathrm{Fe}^{2+}, \mathrm{Fe}^{3+}$, sowie Spurenelemente (wie $\mathrm{Al}, \mathrm{Mn}, \mathrm{Zn}$, As, $\mathrm{Cd}, \mathrm{Cu}, \mathrm{Ni}, \mathrm{Pb}$ ).

Die mit Schwermetallionen belasteten sauren Grubenwässer können beim Ausfluss aus dem Bergwerk in natürliche Gewässer die dort lebenden Tiere und Pflanzen schädigen oder töten und verschlechtern insgesamt die Wasserqualität (Trinkwasser soll nach der Trinkwasserverordnung einen $\mathrm{pH}$-Wert zwischen 6,5 und 9,5 haben und die Schwermetallgrenzwerte nicht überschreiten), sowie die Stabilität des betroffenen Ökosystems. Eine Aufbereitung der sauren Grubenwässer, sowie eine pH-Wert Überwachung ist daher erforderlich [2].

Ein robustes sensorgestütztes EchtzeitÜberwachungssystem würde helfen, die Notwendigkeit und Größenordnung von Aufbereitungsmaßnahmen der Grubenwässer zur pH-Wert-Neutralisation und Schwermetallionenausfällung zu bestimmen und somit die Auswirkungen auf die Umwelt zu minimieren. Ein solches System erfordert allerdings eine möglichst exakte, lokal kontinuierlich einsetzbare und unter den korrosiven Bedingungen stabile Messmethodik.

Aktuell werden vor allem Glaselektroden und Indikatorpapier für die Überwachung des $\mathrm{pH}$-Wertes in sauren Grubenwässern im Bergwerk eingesetzt. Indikatorpapiere müssen manuell angewendet werden und sind daher für eine automatisierte und kontinuierliche Datenaufnahme nicht geeignet. Konventionelle Standard-pHGlaselektroden als potentiometrische Sensoren liefern zwar ein elektrisches Signal und sind somit prinzipiell für automatisierte Systeme einsetzbar. Sie sind aber in sauren Grubenwässern wegen der hohen Konzentrationen an Metall-, Chalkogenid- und Halogenid-lonen zur Bestimmung des $\mathrm{pH}-$ Wertes der Lösungen nicht gut geeignet. Die dünne Glasmembran der $\mathrm{pH}$-Glaselektrode baut sich in Wasser mit hohen Konzentrationen an Mehrfachionen schnell ab und ist auch nur bedingt mechanisch robust. Darüber hinaus verringern Schmutz, Staub und Mikroorganismen im Wasser die Lebensdauer der Elektrode. All dies beeinflusst die Genauigkeit von $\mathrm{pH}$-Glaselektroden in solchen Umgebungen durch starke Drifteffekte und eine beschleunigte Alterung der Elektroden. Damit verbunden sind Nachteile im Hinblick auf große Ungenauigkeiten der $\mathrm{pH}-$ Wertmessungen, ein hoher Materialverschleiß der $\mathrm{pH}-$ Sensoren und ein hoher Wartungsaufwand.

Alternative robuste, miniaturisierbare und integrierbare $\mathrm{pH}-$ Sensoren, die zugleich einen geringeren Wartungsaufwand und eine erhöhte Lebensdauer aufweisen, könnten entscheidend die pH-Wert-Überwachung im Bergwerk verbessern und die Verschleißkosten und die Umweltbelastung reduzieren. Um diesen Anforderungen gerecht zu werden, bieten sich feldeffektbasierte $\mathrm{pH}$-Sensoren [6] an, diese wurden aber bisher noch nicht systematisch für den Einsatz in sauren Grubenwässern untersucht.

Im Rahmen dieser Arbeit wurden daher Versuche mit Elektrolyt-Isolator-Halbleiter-(EIS)-Strukturen durchgeführt, um ihre Eignung zur Überwachung von sauren Grubenwässern zu evaluieren. Die Besonderheit solcher EISStrukturen ist ihr einfacher Aufbau, der im einfachsten Fall aus einer dünnen Metalloxidschicht auf einem Halbleitermaterial besteht und sich für ein schnelles und kostengünstiges Materialscreening eignet. Die Materialien können in gängige feldeffektbasierte Sensoren überführt werden. Das Funktionsprinzip dieser $\mathrm{pH}$-Sensoren ist in der Literatur beschrieben [7]. Es beruht auf der Wechselwirkung des sauren Grubenwassers mit der oberflächennahen $\mathrm{pH}$-sensitiven Metalloxidschicht des Sensors, die die Ausbildung eines $\mathrm{pH}$-abhängigen Oberflächenpotentials nach dem „site-binding-Modell“ verursacht [8]. Dieses Po- 
tential beeinflusst signifikant die Ladungsträgerverteilung in der Halbleiterschicht, was bei einer EIS-Struktur durch eine Verschiebung der Kapazitäts-/Spannungskurven (C/V) detektiert werde kann. Diese Verschiebung erfolgt direkt proportional zur pH-Wert-Änderung.

\section{Experimentelle Arbeiten}

Die für die Versuche genutzten EIS-Strukturen bestehen aus jeweils einer dünnen Metalloxidschicht, die auf einen $<100>$ mit $10^{16}$ Atome $/ \mathrm{cm}^{3}$ Bor p-dotierten $300 \mu \mathrm{m}$ dicken Siliziumwafer mit einem thermischen Oxid von $55 \mathrm{~nm} \pm$ $5 \mathrm{~nm}$ Schichtdicke aufgebracht wurde. Den Rückseitenkontakt bildet eine dünne Aluminiumschicht. Es wurden jeweils drei 1,2 cm x 1,2 cm große Stücke untersucht, die aus dem gleichen Wafer herausgetrennt wurden.

Aufgrund der in der Literatur beschriebenen hohen Sensitivität, Linearität und geringen Hysterese [9] [10] [11] wurden als $\mathrm{pH}$ sensitive Metalloxide $\mathrm{Ta}_{2} \mathrm{O}_{5}$ und $\mathrm{Nb}_{2} \mathrm{O}_{5}$ ausgewählt. Die Beschichtung der sensitiven Schichten erfolgte mittels Magnetronsputtern bei einer Rotation von 5 $\mathrm{U} / \mathrm{min}$ für drei verschiedene Schichtstrukturen mit einer Gesamtschichtdicke von $120 \mathrm{~nm}$. Im Detail wurden reine $\mathrm{Ta}_{2} \mathrm{O}_{5}$-Schichten, $\mathrm{Nb}_{2} \mathrm{O}_{5}$-Schichten, und ein alternierendes Nanolaminat aus abwechselnd $20 \mathrm{~nm}$ dicken $\mathrm{Ta}_{2} \mathrm{O}_{5}$ und $\mathrm{Nb}_{2} \mathrm{O}_{5}$ Schichten mit $\mathrm{Ta}_{2} \mathrm{O}_{5}$ als äußere Schicht abgeschieden. Die Schichten wurden anschließend noch einer RTP (rapid thermal processing)-Temperaturbehandlung unterzogen, um ihre Sensoreigenschaften zu verbessern. $\mathrm{Ta}_{2} \mathrm{O}_{5}$ besitzt laut Literatur [9] [10] [12] die besten $\mathrm{pH}$ sensitiven Eigenschaften in amorpher Form und zudem die Eigenschaft eines Protonenleiters [13], weshalb es als Deckschicht des Nanolaminates verwendet wurde.

Als Analyt wurden Schwefelsäure und Grubenwasserproben genutzt, die aus dem Forschungs- und Lehrbergwerk „Reiche Zeche“ der TU Bergakademie Freiberg entnommen wurden. Die Probeentnahmestandorte mit den Grubenwasserflussrichtungen sind in Abb. 1 dargestellt.

Nach der Probennahme wurden die Proben hinsichtlich ihrer Leitfähigkeit, ihres Redoxpotentials, dem $\mathrm{pH}-$ Wert, sowie der Durchflussmenge für den jeweiligen Probennameort charakterisiert. In Tabelle 1 sind die Messparameter der Probennamestellen dargestellt.

Tabelle 1: Messparameter $\mathrm{pH}-$ Wert, Leitfähigkeit und Redoxpotential der Realmessproben von sauren Grubenwässern aus der Reichen Zeche, Freiberg.

\begin{tabular}{|l|l|l|l|l|}
\hline Proben & $\begin{array}{l}\text { Probeentnahmeort } \\
\text { In der Reichen Zeche }\end{array}$ & $\begin{array}{l}\text { pH-Wert } \\
\text { Glaselektrode }\end{array}$ & $\begin{array}{l}\text { mittlere } \\
\text { Leitfăhigkeit }\end{array}$ & $\begin{array}{l}\text { Redox- } \\
\text { potential }\end{array}$ \\
\hline Probe \#1 & BHMZ (WHSt13) & 2,71 & $4,74 \mathrm{mS}$ & $530 \mathrm{mV}$ \\
\hline Probe \#2 & HGST-PSQW & 7,16 & $765 \mu \mathrm{S}$ & $330 \mathrm{mV}$ \\
\hline Probe \#3 & HGSt 14 & 7,51 & $2,78 \mathrm{mS}$ & $600 \mathrm{mV}$ \\
\hline
\end{tabular}

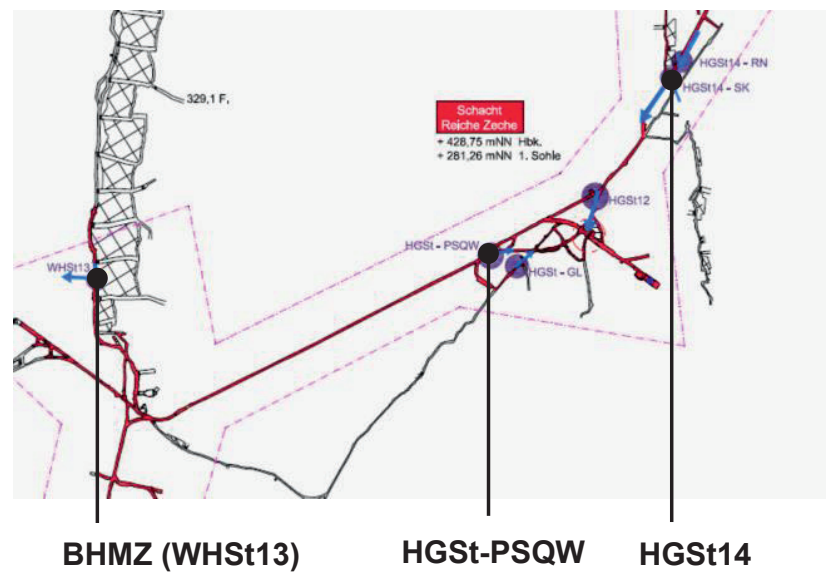

Abb. 1: Grubenwasser Probeentnahmestandorte aus dem Forschungs- und Lehrbergwerk „Reiche Zeche“ (1. Sohle) der TU Bergakademie Freiberg, die Grubenwasserflussrichtungen sind mit blauen Pfeilen gekennzeichnet. Die Durchflussmenge an den Standorten schwankt über das Jahr je nach niederschlagsbedingten Wassereintrag von 1,61 L/s (HGSt14) bis 0,021 L/s (WHSt 13) [3].

Der verwendete Messaufbau ist in Abb. 2 dargestellt. Zur Messung wird die zu messende EIS-Struktur in eine Durchflussmesszelle mit integrierten Messelektroden und Fluidikzuführungsschläuchen eingespannt. Über eine $\mathrm{Ag} / \mathrm{AgCl}$-Referenzelektrode $(3 \mathrm{M} \mathrm{KCl}$ ), die den Vorderseitenkontakt durch das Grubenwasser herstellt, sowie eine Kupferplatte, die im Kontakt mit der leitfähigen Rückseite der EIS-Struktur steht, wird der elektrische Messkreis geschlossen. Die verwendeten Elektroden sind durch geschirmte Kabel an ein LCR-Meter (HP 4284A) angeschlossen, das dann die C/V-Kurven aufnimmt. Zur Bereitstellung, Mischung und Dosierung der GrubenwasserProben, sowie der Spülung des Systems, wurden Dosierpumpen und ein 6-Wege-Ventil verwendet. Das gesamte Messsystem wird über ein LabView-Programm gesteuert.

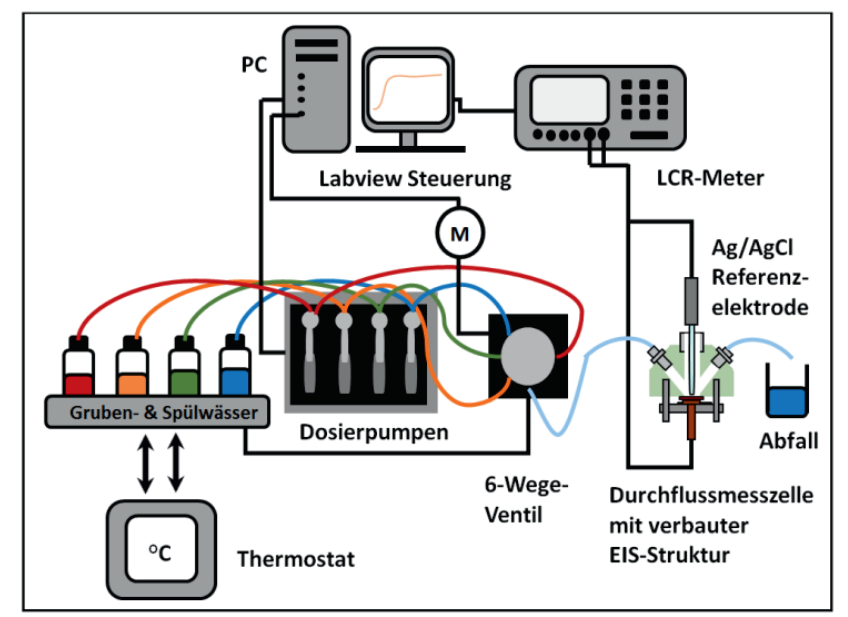

Abb.2: Schematische Darstellung des Messaufbaus 


\section{Ergebnisse und Diskussion}

$\mathrm{Da} \mathrm{Nb}_{2} \mathrm{O}_{5}$ eine Vielzahl an Kristallphasen mit der stabilen TT-, T-, M- und H-Phase [14] besitzt, wurde zunächst untersucht, welche Kristallphase und damit Temperaturbehandlung für einen $\mathrm{Nb}_{2} \mathrm{O}_{5}-\mathrm{pH}$-Sensor am besten geeignet ist. Dazu wurden die Sensitivität der jeweiligen Probe im $\mathrm{pH}$-Bereich von 3 bis 11 sowie die zugehörige Linearität des Messsignals bestimmt. Weiterhin wurde die Hysterese bei $\mathrm{pH} 3$ ermittelt.

In Abb. 3 sind die ermittelten Sensorparameter in Abhängigkeit der RTP-Temperaturbehandlung dargestellt.

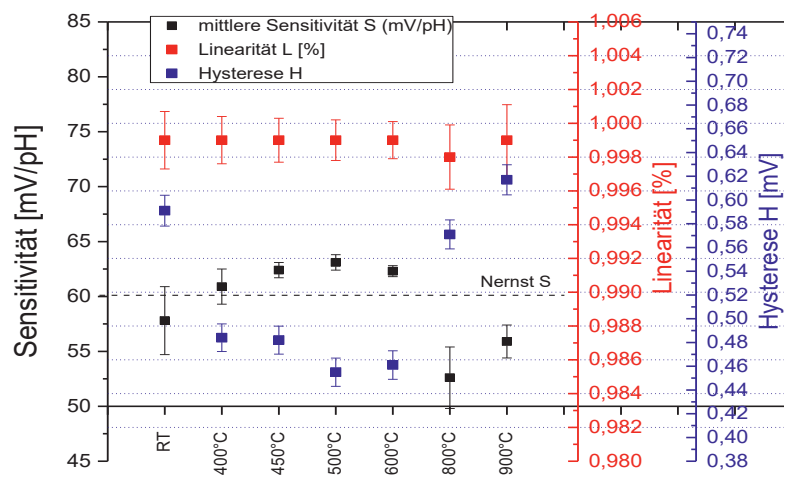

Abb.3: Ermittelte Sensitivitäts-, Linearitäts- und mittleren Hysterese- Werte für eine $120 \mathrm{~nm} \mathrm{Nb}_{2} \mathrm{O}_{5}$-Schicht (Beschichtung bei Raumtemperatur) und anschließender RTP-Temperaturbehandlung von $400^{\circ} \mathrm{C}$ bis $900^{\circ} \mathrm{C}$.

Die niedrigste Hysterese bei hoher Sensitivität und Linearität wurde für eine Temperatur von $500{ }^{\circ} \mathrm{C}$ für $\mathrm{Nb}_{2} \mathrm{O}_{5}$ gemessen und daher ausgewählt. Bei $500{ }^{\circ} \mathrm{C}$ liegt $\mathrm{Nb}_{2} \mathrm{O}_{5}$ gemäß durchgeführter XRD-Messungen in der amorphen Phase mit geringen TT-Phasenanteilen vor. $\mathrm{Ta}_{2} \mathrm{O}_{5}$ wurde ebenfalls bei $500{ }^{\circ} \mathrm{C}$ RTP behandelt und liegt bei dieser Temperatur noch amorph vor.

Um die generelle Verwendbarkeit des Messprinzips für saure Grubenwässer zu demonstrieren und Auskunft über die Signaländerungen bei geringen $\mathrm{pH}$-Wert-Änderungen zu geben, wurde ausgehend von einer realen Grubenwasserprobe mit pH 7,51 schrittweise 10\%ige Schwefelsäure zutitriert, um eine breite Verteilung von $\mathrm{pH}$-Werten saurer Grubenwässer zu simulieren. Nach jedem Titrationsschritt wurden der $\mathrm{pH}$-Wert des Messelektrolyten mit einer konventionellen $\mathrm{pH}$-Glaselektrode bestimmt und jeweils $5 \mathrm{CV}$ Kurven aufgezeichnet, aus denen dann jeweils ein Mittelwert und die Standardabweichung der Sensorantwort bestimmt wurden.

Die Sensorantwort des $\mathrm{Nb}_{2} \mathrm{O}_{5}$-Sensors in Abhängigkeit der zugegebenen Menge an Schwefelsäure zur Simulation verschieden saurer Grubenwässer ist in Abb. 4 dargestellt. Wie zu erwarten war nimmt der $\mathrm{pH}-$ Wert mit zunehmenden Schwefelsäuregehalt ab. Das Sensorsignal folgt diesem Verlauf in erster Näherung linear mit einer Steigung von etwa $63,5 \mathrm{mV} / \mathrm{pH}$ und einem $\mathrm{R}^{2}$-Wert von 0,995 über den betrachteten Bereich von $\mathrm{pH}$ 7,3 bis $\mathrm{pH}$ 1,6.

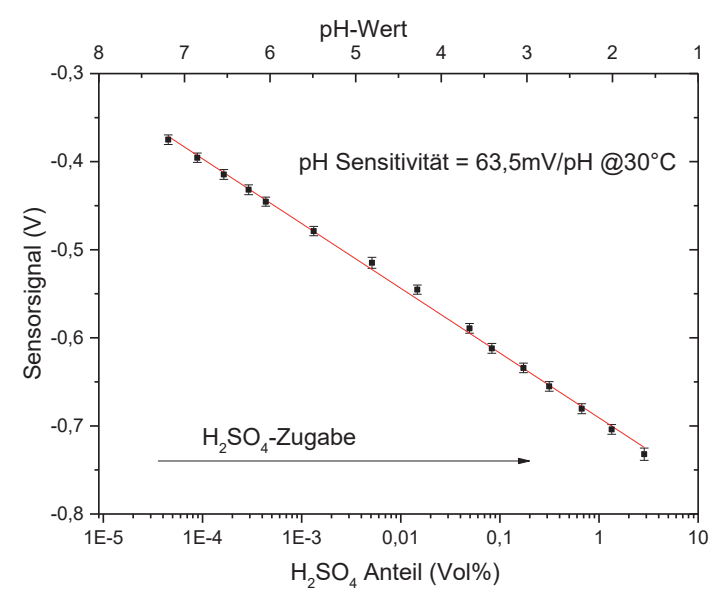

Abb.4: Verlauf des gemessenen Sensorsignals der $\mathrm{Nb}_{2} \mathrm{O}_{5}-$ EIS-Struktur mit zunehmenden $10 \%$ tigen $\mathrm{H}_{2} \mathrm{SO}_{4}$-Anteil bei $30{ }^{\circ} \mathrm{C}$.

Die Standardabweichung an den aufgenommenen Messpunkten beträgt weniger als $0,7 \mathrm{mV}$, was ca. $0,01 \mathrm{pH}$ Schritten entspricht. Damit sind alle simulierten $\mathrm{H}_{2} \mathrm{SO}_{4}-$ Anteile an sauren Grubenwässern klar zu unterscheiden. Um zu demonstrieren, dass sich die EIS-Sensoren mit den ausgewählten Sensormaterialien auch für die Messung der $\mathrm{pH}-$ Werte realer unterschiedlich saurer Grubenwasserproben nutzen lassen können, wurde die Durchflussmesszelle zunächst mit der realen Grubenwasserprobe mit $\mathrm{pH}$ 7,51 befüllt und nach zwei Minuten eine C/V-Kurve gemessen. Anschließend wurden verschiedene saure Grubenwasser Proben ( $\mathrm{pH}=2,71 ; \mathrm{pH}=7,16 ; \mathrm{pH}=7,51$ ) in die Messzelle gepumpt und jeweils zwei Minuten zur Quellschichtausbildung sowie Messlösungsberuhigung gewartet. Dann wurde wieder eine C/V-Kurve gemessen und die Flachbandspannungsänderung registriert. Diese Prozedur wurde zyklisch wiederholt um eine mögliche Hysterese beim Wechsel von einer Grubenwasserprobe zur nächsten auszuschließen. In Abb. 5 sieht man beispielhaft das Verhalten einer $\mathrm{Ta}_{2} \mathrm{O}_{5}-\mathrm{Nb}_{2} \mathrm{O}_{5}-\mathrm{Nanolaminat-}$ EIS-Struktur.

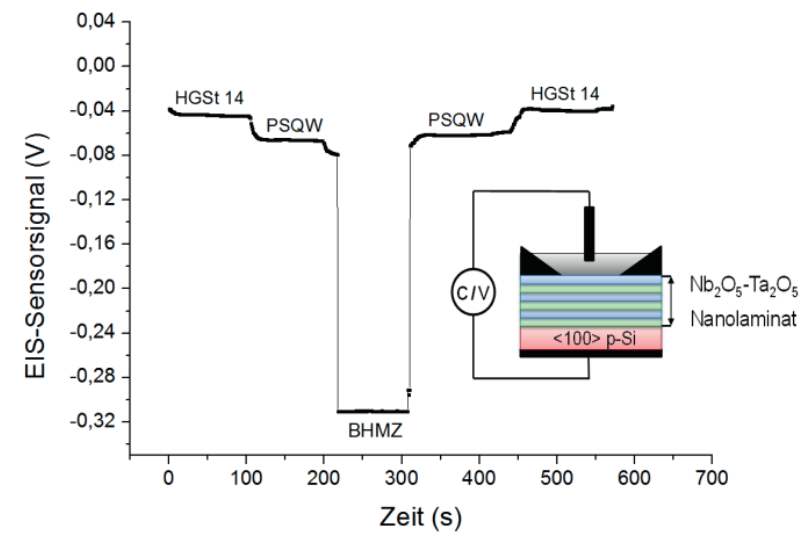

Abb. 5: Beispiel eines Sensorsignalverlaufes bei $30^{\circ} \mathrm{C}$ bei Beaufschlagung mit Realproben saurer Grubenwässer. 
Insert: Sensoraufbau mit $\mathrm{Ta}_{2} \mathrm{O}_{5}-\mathrm{Nb}_{2} \mathrm{O}_{5}-\mathrm{Nanolaminat}$ als sensitive Schicht.

Der Übergang von einem stabilen Messsignal zum nächsten erfolgte dabei in ca. $10 \mathrm{~s}$. Die $10 \mathrm{~s}$ entsprechend der Ansprechzeit für den hier verwendeten Durchflussmesszellenaufbau. Ohne vorherigen Flüssigkeitskontakt und damit verbundener Quellschichtausbildung (Trockenzustand des EIS-Sensors) würde sich die Ansprechzeit entsprechend verlängern. Außerdem ist keine signifikante Messrichtungsabhängigkeit bezüglich der Richtung der $\mathrm{pH}$-Wertänderung und damit eine zu vernachlässigende Hysterese zu beobachten. Daher könnten diese Sensoren auch über längere Zeiträume mit schwankendem Grubenwasserdurchfluss bei dynamischen Zusammensetzungen und $\mathrm{pH}-$ Werten verwendet werden.

Der Vergleich der Ergebnisse der drei ausgewählten Sensorstrukturen sind in Abb. 6 dargestellt. Alle drei Materialien zeigen eine lineare Abhängigkeit des Sensorsignals von den Grubenwässern mit verschiedenem pH-Wert. Außerdem wurde an den jeweiligen Messpunkten für alle Sensormaterialien eine geringe Standardabweichung des Sensorsignals von ca. $1 \mathrm{mV}(<0,02 \mathrm{pH})$ ermittelt. Dies spricht für ein stabiles Messsignal und somit für einem robusten $\mathrm{pH}$-Sensor. Die Aussagkraft dieser Messung ist jedoch mit Vorsicht zu betrachten, da mit den drei Grubenwasser-Proben nur drei Messpunkte betrachtet wurden.

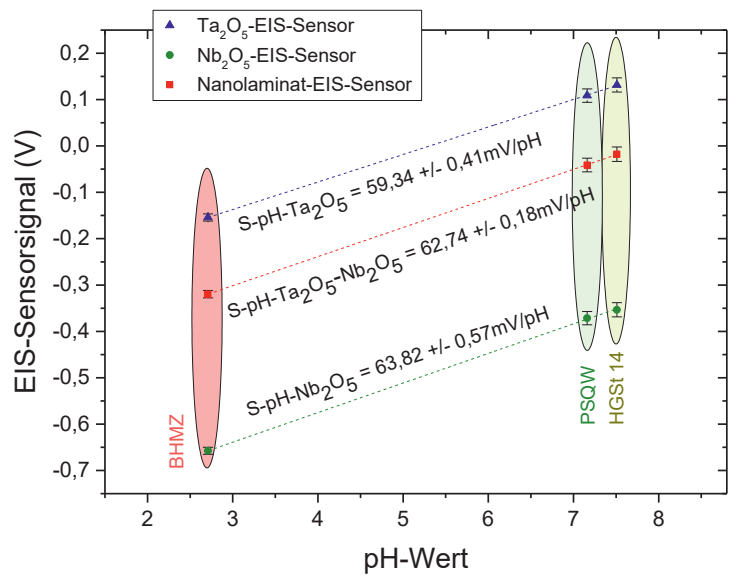

Abb.6: Korrelation der Sensorantwort für die 3 verwendeten Sensortypen mit dem $\mathrm{pH}$-Wert der Realproben.

Letztlich sollte die Langzeitstabilität der Sensoren überprüft werden. Dafür wurden EIS-Strukturen mit den drei Sensormaterialien über einen Zeitraum von 4 Monaten in einem simulierten sauren Grubenwassermillieu mit $10 \%$ iger Schwefelsäure gelagert und mehrfach im Abstand von jeweils 30 Tagen vermessen. Aus den Messungen wurden die Sensitivitäts-, Linearitäts- und Hysteresewerte bestimmt. Diese sind in Abb. 7 dargestellt.

Alle drei Sensoren haben über die Dauerbeanspruchungszeit erfolgreich ihre grundlegende Sensorfunktion erhalten, wobei bei allen drei Sensoren ähnliche Tendenzen festgestellt wurden. Bei allen Materialien wurden eine geringe Abnahme der Linearität, eine geringe Zunahme der Hysterese und eine geringfügige Verschlechterung der Sensitivität verzeichnet.
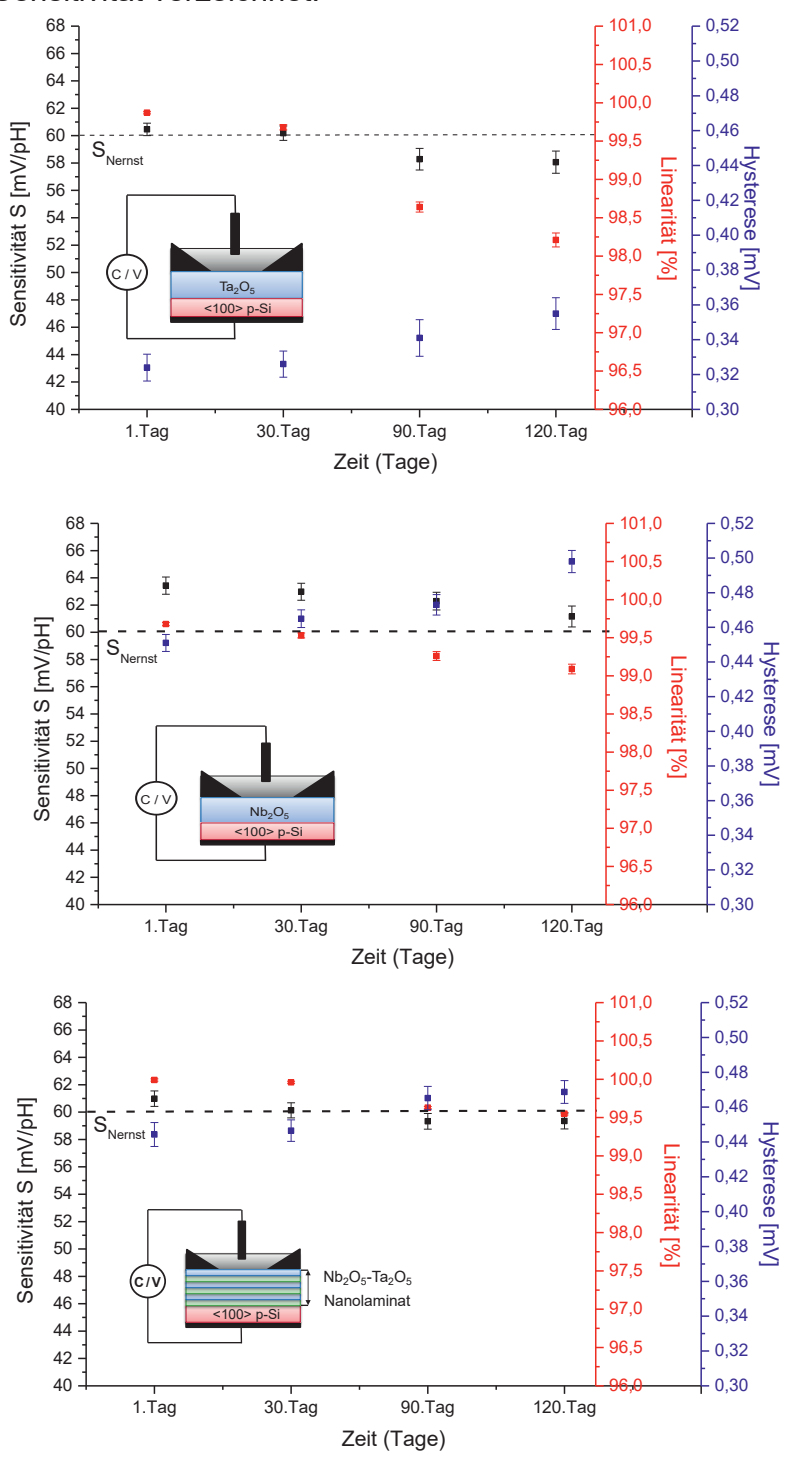

Abb.7: Korrelation der Sensitivität, Linearität und mittleren Hysterese für die 3 Sensormaterialien: $\mathrm{Ta}_{2} \mathrm{O}_{5}-$ Monolayer (oben), $\mathrm{Nb}_{2} \mathrm{O}_{5}-$ Monolayer (Mitte) und dem $\mathrm{Ta}_{2} \mathrm{O}_{5}-\mathrm{Nb}_{2} \mathrm{O}_{5}-$ Nanolaminat (unten) in $10 \%$ iger $\mathrm{H}_{2} \mathrm{SO}_{4}$ über einem Zeitraum von 120 Tagen bei $30^{\circ} \mathrm{C}$.

$\mathrm{Ta}_{2} \mathrm{O}_{5}$ zeigte die geringsten Hysterese-Werte von ca. 0,32 $\mathrm{mV}$ bis $0,34 \mathrm{mV}, \mathrm{Nb}_{2} \mathrm{O}_{5}$ zeigte die höchsten HystereseWerte von ca. $0,45 \mathrm{mV}$ bis $0,49 \mathrm{mV}$ und die Nanolaminatverbundstruktur aus $\mathrm{Ta}_{2} \mathrm{O}_{5}$ und $\mathrm{Nb}_{2} \mathrm{O}_{5}$ zeigte eine mittlere Hysterese von $0,42 \mathrm{mV}$ bis $0,47 \mathrm{mV}$. Die Linearitätswerte wurden bei allen Sensoren am ersten Messtag mit ca. $99,9 \%$ bestimmt. Sie nahmen beim $\mathrm{Ta}_{2} \mathrm{O}_{5}$ auf $98,2 \%$ ab, beim $\mathrm{Nb}_{2} \mathrm{O}_{5}$ erfolgte eine Abnahme auf $99,2 \%$. Die Nanolaminatverbundstruktur aus $\mathrm{Ta}_{2} \mathrm{O}_{5}$ und $\mathrm{Nb}_{2} \mathrm{O}_{5}$ zeigte nur eine sehr geringe Abnahme auf $99,6 \%$ bis zum 120. Tag. Die Sensitivitätswerte lagen zu Beginn der Messung bei etwa $60 \mathrm{mV} / \mathrm{pH}$ und zeigten nur leichte Abnahmen über die Messdauer, wobei auch hier die Nanolaminat-Struktur die geringste Abnahme zeigte. Insgesamt konnte somit für alle drei Sensorstrukturen ein robustes stabiles Sensor- 
signal über die Beanspruchungszeit von 120 Tagen festgestellt werden. Die $\mathrm{Ta}_{2} \mathrm{O}_{5}-\mathrm{Nb}_{2} \mathrm{O}_{5}-\mathrm{Nanolaminat} \mathrm{EIS}$ Struktur zeigt von den drei EIS-Strukturen die höchste Signalstabilität und Robustheit.

\section{Zusammenfassung und Ausblick}

Das Ziel dieser Arbeit war es, feldeffektbasierte $\mathrm{pH}$ Sensoren auf ihre Verwendbarkeit für die lokale Überwachung des $\mathrm{pH}-$ Wertes saurer Grubenwässer hin zu untersuchen.

Für diesen Zweck wurden $\mathrm{pH}$ sensitive EIS-Strukturen hergestellt, die sensitiven Materialien $\left(\mathrm{Ta}_{2} \mathrm{O}_{5}\right.$ und $\left.\mathrm{Nb}_{2} \mathrm{O}_{5}\right)$, hinsichtlich ihrer Struktur optimiert und anhand von realen Grubenwasserproben getestet. Die untersuchten $\mathrm{pH}$ Sensoren zeigen eine Sensitivität nahe dem theoretischen Limit, sowie eine hohe Reproduzierbarkeit der Messergebnisse mit geringen Schwankungen. Des Weiteren konnte gezeigt werden, dass sich durch die Schichtung der $\mathrm{pH}$ sensitiven Materialien in Nanolaminatverbundstrukturen, die Stabilität und Lebensdauer von feldeffektbasierten $\mathrm{pH}$ Sensoren erhöhen lässt.

Feldeffektbasierte $\mathrm{pH}$-Sensoren sind somit für den Einsatz zur Überwachung von sauren Grubenwässern geeignet und ihre Integration in in-situ-Überwachungssysteme im Bergwerk könnte helfen unerwünschte Korrosionsprozesse an Bauwerken und Maschinen vor Ort, sowie den Austritt von stark schwermetallhaltigen sauren Wässern durch eine gezielte Nachbehandlung umzusetzen. Damit wäre ein wichtiger Schritt hin zu einem umwelt- und materialschonenderen Bergbau gemacht.

Das zukünftige Ziel ist es, ein Gesamtsystem aus feldeffektbasiertem $\mathrm{pH}$-Sensor, Referenzelektrode, sowie einem integrierten Temperatursensor zur internen Kalibrierung zu entwickeln und zu optimieren, sodass in-situ-Versuche im Forschungs- und Lehrbergwerk „Reiche Zeche“ erfolgen können. Ebenfalls angedacht ist die Überführung der getesteten Materialien in ionensensitive Feldeffekttransistoren (ISFET) oder extended-gate Feldeffekttransistoren (EGFET) [15], die eine direkte Implementation in integrierte Schaltkreise (ICs) und Sensorsystemen ermöglichen.

\section{Literatur}

[1] SIMATE, G. S.; NDLOVU, S.: Acid mine drainage: Challenges and opportunities, Research Papers of School of Chemical and Metallurgical Engineering, University of the Witwatersrand, Johannesburg, South Africa, 2014

[2] KEFENI, K. K.; MSAGATI, T. A.M.; MAMBA, B. B.: Acid mine drainage: Prevention, treatment options, and resource recovery: A review. In: Journal of Cleaner Production, Nr. 151, S. 475 - 493, 2017

[3] DULAMJAV, U.: Zeitlich und räumlich aufgelöste Analyse ausgewählter Stoffströme im Grubenfeld des Forschungs- und Lehrbergwerkes der TU Bergakademie Freiberg. Technische Universität Bergakademie Freiberg, Studienarbeit, 2017

[4] PIERRE LOUIS, A.-M.; YU, H.; SHUMLAS, L.; VAN AKEN, B.; SCHOONEN, M. A. A.; STRONGIN, D. R.:
Effect of Phospholipid on Pyrite Oxidation and Microbial Communities under Simulated Acid Mine Drainage (AMD) Conditions. In: Environ. Sci. Technol. 49, S. 7701-7708, 2015

[5] MISCHO, H.; ZHITENEVA, V.; BRUNE, J.: Water quality of Reiche Zeche teaching mine. Freiberg, Saxony, Germany. In: Conference: SME Annual Meeting and Mining Engineering Conference, At: Phoenix, Arizona, USA, 2016

[6] SCHÖNING, M.J.: "Playing around" with Field-Effect Sensors on the Basis of EIS Structures, LAPS and ISFETs. In: Sensors 5 (2005), Nr. 3, S.126-138

[7] GÜTH, F.; ARKI, P.; JOSEPH, Y.: Feldeffektba sierte $\mathrm{pH}$-Sensoren für die Überwachung von Kühl schmierstoffen. 12.Dresdner Sensor Symposium, 2015

[8] YATES, D.E.: LEVINE, S.; HEALY, T. W.: Site-binding Model of the Electrical Double Layer at the Oxide/Water Interface. In: J. Chem. Soc. Faraday Transactions 1 (1974), Nr. 70, S.1807-1818

[9] MIKOLAJICK, T.; KÜHNHOLD, R.; RYSSEL, H.: The $\mathrm{pH}$-sensing properties of tantalum pentoxide films fabricated by metal organic low pressure chemical vapor deposition. In: Sensors and Actors B: Chemical 44 (1997), Nr. 1-3, S.262-267

[10] BOUSSE, L.; MOSTARSHED, S.; VAN SHOTT, B.; DE ROOIJ, N. F.: Comparison of the Hysteresis of Ta2O5 and Si3N4 pH-Sensing Insulators. In: Sensors and Actuators B 17, S. 157-164, 1994

[11] MIKOLAJICK, T.: Feldeffektsensoren zur pH-WertMessung und als Transducer für Biosensoren. In: Erlanger Berichte Mikroelektronik, Shaker Verlag, TU Erlangen-Nürnberg, Dissertation, 1996

[12] GRIMMEL, P.: Ionensensitive Ta2O5- Feldeffekttransistoren: Aufbau, Optimierung und Einsatz in der Biosensorik. Eberhard-Karls-Universität Tübingen, Fakultät für Chemie und Pharmazie, Dissertation, 1991

[13] TAJIMA, K.; YAMADA, Y.; BAO, S.; OKADA, M.; YOSHIMURA, K.: Proton conductive tantalum oxide thin film deposited by reactive DC magnetron sputtering for all-solid-state switchable mirror. In: J. Phys.: Conf. Ser. (2008), 100 (8), 82017

[14] SCHÄFER, H.; GRUEHN, R.; SCHULTE, F.: The modifications of niobium pentoxide. In: Angewandte Chemie Int. Ed. Engl., S.40-52, 1966

[15] KAO, C.-H.; CHEN, H.; KUO, L.-T.; WANG, J.-C.; CHEN, Y.-T.; CHU, Y.-C.; CHEN, C.-Y.; LAI, C.-S.; CHANG, S. W.; CHE WEI, C.: Multi-analyte biosensors on a CF4 plasma treated Nb2O5-based membrane with an extended gate field effect transistor structure. In: Sensors and Actuators B: Chemical 2014, Nr. 194, S. $419-426,2014$

\section{Danksagung}

Die Autoren danken den Verantwortlichen für den Zugang zum Forschungs- und Lehrbergwerk „Reiche Zeche“ der TU Bergakademie Freiberg, sowie für die Möglichkeit der Probenname vor Ort. 